\title{
Il sole e il bene. \\ Funzione e limiti dell'analogia in Resp. VI 505a-509b
}

\author{
The Sun and the Good. \\ Functions and limits of the analogy in Resp. VI 505a-509b
}

\author{
FRANCESCO FRONTEROTTA \\ Università La Sapienza di Roma \\ francesco.fronterotta@uniroma1.it
}

Recibido: 03/02/2017 - Aceptado: 14/09/2017

DOI: https://doi.org/10.20318/fons.2017.3855

\begin{abstract}
Riassunto. La presenza dell'analogia nel libro VI della Repubblica di Platone, concepita come rapporto, orizzontale o verticale, di semplice uguaglianza funzionale tra ambiti eterogenei, dischiude promettenti scenari di comprensione sullo statuto e la funzione dell'idea del bene in sé e in relazione ai suoi stessi "prodotti”. In particolare, l’immagine del Sole come "figlio" del bene non implica alcuna ammissione da parte di Platone di una generazione o di una derivazione integrale del primo dal secondo, così come non suggerisce una derivazione integrale delle cose sensibili dalle idee intellegibili.
\end{abstract}

Parole Chiave: Repubblica, bene, Sole, analogia, produttore/prodotti, idee, realtà sensibile

\begin{abstract}
The presence of analogy in Book VI of Plato's Republic, conceived as a relationship, horizontal or vertical, of functional equality between heterogeneous domains, offers promising options to understand the status and function of the good itself and in relation to its own "products". In particular, the image of the Sun as the "son" of the good does not imply that Plato admits an integral generation or a derivation of the first from the second, nor does it suggest an integral derivation of sensible things from intelligible ideas.
\end{abstract}

Keywords: Republic, good, Sun, analogy, productor/products, ideas, sensible reality

ПНГН/F O NS II (2017), 109-122 ISSN 2445-2297 www.uc3m.es/pege
F. FronterotTA, Il sole e il bene. Resp. VI 505a-509b DOI https://doi.org/10.20318/fons.2017.3855 
L'analogia dell'essere nella storia della filosofia è, in generale e un po' grossolanamente, lo strumento tramite il quale giungere ad acquisire una conoscenza e a formulare un discorso intorno a un principio primo, eventualmente divino, a partire dai suoi prodotti, assumendo che, poiché sussiste una qualche forma di rapporto fra i prodotti e il loro produttore, possiamo accedere alla conoscenza del produttore attraverso la conoscenza dei suoi prodotti. A questo fine occorre che l'analogia come tale sia concepita come nozione non orizzontale, che implichi una semplice uguaglianza di rap-

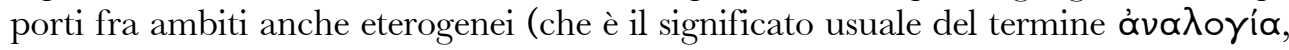
innanzitutto nel senso di "proporzione" matematica, nella lingua greca classica), perché ciò porterebbe, per un verso, a collocare il produttore e i suoi prodotti sullo stesso piano (se la relazione analogica è orizzontale) e, per altro verso, a non acquisire di fatto nessuna conoscenza del produttore a partire dai suoi prodotti (se produttore e prodotti appartengono ad ambiti eterogenei); occorre invece concepire l'analogia come una relazione verticale, perché il produttore trascende i suoi prodotti ed è loro superiore, e in qualche modo derivativa e causale, non fosse altro che intendendo tale causalità come esemplare, perché vi sia una qualche forma di comunanza o somiglianza fra produttore e prodotti. In altre parole, poiché non può darsi che l'analogia fra produttore e prodotti induca a collocarli sullo stesso piano, bisogna che essa si tra-

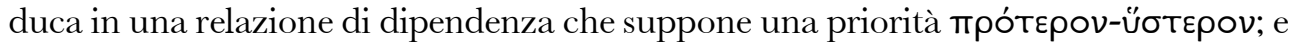
perché l'analogia apra l'accesso a una più o meno ampia conoscenza del produttore a partire dai prodotti, bisogna che questi conservino una traccia di quello, da cui devono perciò derivare causalmente 1 .

La tradizione medievale ha voluto individuare il nucleo teorico di questa dottrina in Aristotele, particolarmente nella sua tesi della molteplicità dei sensi dell'essere, né sinonimi né semplicemente omonimi, cioè nella celebre relazione mpòs Ěv tramite la quale Aristotele caratterizza la relazione ad unum dei diversi sensi dell'essere secondo le categorie al senso primario della sostanza. Pierre Aubenque, fra gli altri, ha argomentato l'illegittimità di una simile operazione esegetica, in primo luogo perché, quale che sia effettivamente la posizione di Aristotele, egli non impiega mai nell'ambito della sua dottrina dei sensi dell'essere il termine "analogia", che, anzi, conserva nel corpus il senso matematico di "proporzione”, ossia di uguaglianza di rapporti, di per sé inapplicabile al caso della relazione fra i diversi sensi dell'essere e la sostanza (tale relazione non implicando un'uguaglianza di rapporti del tipo: $\mathrm{a}: \mathrm{b}=\mathrm{c}: \mathrm{d}$ ecc., ma una diversità di rapporti in riferimento a un termine primo e identico del tipo: $\mathrm{a}: \mathrm{b}=\mathrm{a}: \mathrm{c}=\mathrm{a}: \mathrm{d}$ ecc.); in secondo luogo, e soprattutto, perché la relazione che Aristotele pone fra i sensi dell'essere secondo le categorie e il primo di essi, la sostanza, non pare caratterizzata

\footnotetext{
${ }^{1}$ Sulla questione dell'analogia dell'essere si possono vedere, da un punto di vista storico-filosofico come da un punto di vista più propriamente teoretico, LYTTKENS 1952, MELCHIORRE 1996 e soprattutto COURTINE 2005. In riferimento alla costituzione metafisica di questa nozione nel corso della storia del platonismo antico, cfr. in generale KRÄMER 1967.
} 
da tratti né "proporzionali”, in qualunque accezione del termine, né in alcun modo derivativi o causali. Tale interpretazione presupporrebbe invece, secondo Aubenque, una "platonizzazione" di Aristotele, basata sulla lettura di alcuni testi di Platone mediata naturalmente dal neoplatonismo e depositata particolarmente nei commentari neoplatonici ad Aristotele: di qui, attraverso cioè il ricorso a Platone e alla tradizione platonica antica e tardo-antica, sorgerebbe quella lettura onto-teologica della metafisica aristotelica, in gran parte incentrata appunto sull'analogia entis, che si pone al centro del dibattito scolastico, poi moderno e contemporaneo intorno alla Metafisica ${ }^{2}$. Cercherò di esaminare in questo contributo, tralasciando un esame lessicale delle occorrenze del termine ớva $\lambda$ oyía e dei suoi derivati nei dialoghi di Platone, come pure, nel suo insieme, una ricostruzione della concezione platonica dell' "analogia" 3 , la seconda parte della tesi di Aubenque, vale a dire appunto l'origine in qualche senso platonica di questa deformazione (in primo luogo neoplatonica) della nozione di "analogia", con riferimento all'unico passo pertinente del corpus, vale a dire alle pagine del libro VI della Repubblica in cui viene proposta la celebre analogia fra il Sole e il bene.

Nel libro VI della Repubblica, a partire da 505a, Socrate è indotto dal suo interlocutore a esprimersi sulla natura dell'idea del bene, concepita come la «conoscenza

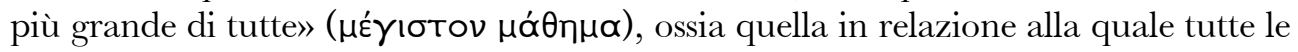
altre cose e tutte le altre discipline divengono realmente utili e vantaggiose. Non è certo possibile considerare qui la lunga sezione del dialogo che, fino a $509 \mathrm{~d}$, affronta il tema delicatissimo ed estremamente controverso dello statuto e della funzione del bene, di cui si giunge a sostenere (508e; 509b) che «garantisce la verità agli oggetti conosciuti e a chi conosce la facoltà di conoscere», che è «causa di scienza e verità ... ma diverso e ancor più bello di esse» e che «da esso gli oggetti di conoscenza traggono l'essere e l'essenza, pur non essendo il bene un'essenza, ma ponendosi ancora al di là dell'essenza per dignità e potenza» ${ }^{4}$. Si tratta notoriamente di uno dei luoghi più enigmatici e dibattuti del corpus platonico che conviene lasciare nel suo insieme da parte, per concentrarsi piuttosto esclusivamente sulle modalità e i limiti che lo stesso Socrate stabilisce per il suo discorso intorno all'idea del bene . Dapprima, infatti, Socrate pre-

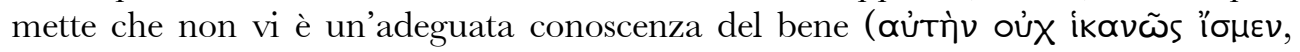

${ }^{2}$ Cfr. Aubenque 2013, pp. 198-206, e Aubendue 1978. Si vedano poi Berti 1984 e 1987, nonché la dettagliatissima ricostruzione d'insieme di COURTINE 2005, pp. 153-229, che è assai più prudente nel negare tout court la radice aristotelica della questione dell'analogia dell'essere.

${ }^{3}$ Ho presentato questo esame lessicale, e tentato di ricavarne i tratti essenziali della concezione pla-

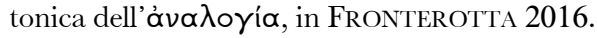

${ }^{4}$ Ricordo soltanto, a mo' di introduzione alla spinosissima questione della natura e della funzione dell'idea del bene nella Repubblica, VEGETTI 2003 e FERRARI 2003b. Il problema è affrontato, in una prospettiva filosofica più ampia, da FerBer 2015.

${ }^{5}$ È stato discusso in particolare, a questo proposito, se Socrate si spinga a esporre nel suo discorso delle opinioni intorno all'idea del bene oppure se tali opinioni riguardino soltanto il "figlio" del bene, 
505a5-6), come mostra la grande varietà di opinioni erronee in proposito, per ribadire tuttavia poco oltre (506a) che tale conoscenza è invece indispensabile in primo luogo per coloro i quali sono destinati al governo della $\kappa \alpha \lambda \lambda i$ mо $\lambda ı s$, per i filosofi, perché, «prima di conoscere il bene, nessuno potrà adeguatamente conoscere quelle cose» che

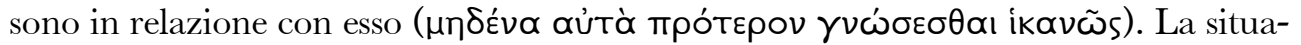
zione attuale degli interlocutori del dialogo non è tuttavia quella dei filosofi giunti al culmine della loro formazione, sicché Socrate si trova nell'imbarazzante condizione

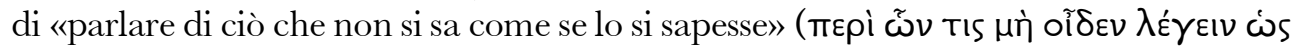
Eỉóta, 506c2-3) e ciò appunto nella misura in cui egli teme di «non essere competente» (oủX oĩos, 506d6) intorno al bene. Di conseguenza, non disponendo della conoscenza del bene, Socrate acconsente a manifestare soltanto la sua opinione su un'immagine di quello, che può dunque essere considerata come «il figlio del bene e

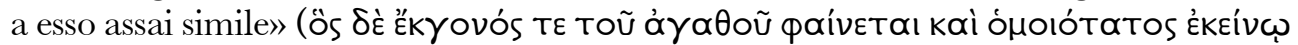

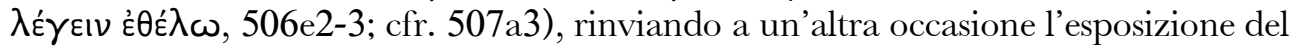
padre, in confronto alla quale l'esame attuale rappresenta soltanto una sorta di interesse rispetto al capitale totale. Il "figlio" del bene è poco dopo (508a-b) identificato con il Sole, che è nel mondo sensibile, in virtù della luce che emette, la causa della vista e della sua capacità di vedere gli oggetti visibili, essendo così stabilita una certa somiglianza fra la vista e il Sole, che pure non coincide né con la vista, che può però assumerlo come proprio oggetto di visione, né con gli oggetti visibili. è precisamente a questo titolo che Socrate individua nel Sole «il figlio del bene» (тòv Toũ áy $\alpha \theta$ oũ

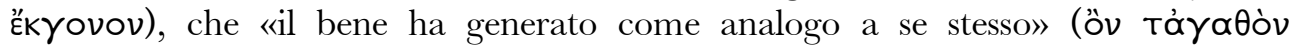

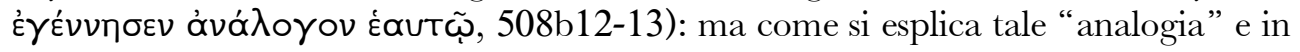
cosa consiste esattamente? La risposta è subito fornita: «come sul piano intellegibile il bene sta all'intelletto e agli intellegibili, così sul piano visibile il Sole sta alla vista e agli

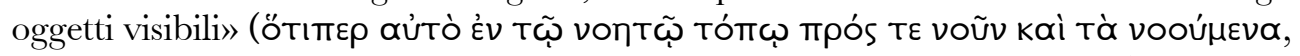

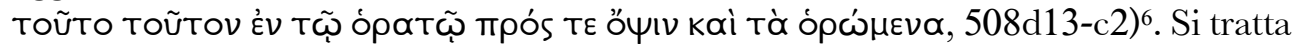
perciò di ammettere una "proporzione", cioè un'identità di rapporto, da una parte, fra il bene, l'intelletto e gli intellegibili e, dall'altra, fra il Sole, la vista e gli oggetti visibili: come il Sole, in quanto fonte di luce, è condizione della visione per la vista e della visibilità per gli oggetti visibili, ponendosi aggiuntivamente come simile alla vista e come suo oggetto di visione, ma non coincidente con essa, così il bene, in quanto fonte di verità, dovrà rappresentare la condizione della conoscenza per l'intelletto e della conoscibilità per gli intellegibili, ponendosi inoltre come simile all'intelletto e

subito oltre introdotto come sua "immagine", e si limitino pertanto a toccare una copia sensibile del bene: la scelta non è indifferente rispetto all'interpretazione della concezione epistemologica di Platone. Si vedano rispettivamente, in proposito, FERRARI 2003a, pp. 395-399, e FrONTEROTTA 2007, pp. 129-133.

${ }^{6}$ Per una presentazione d'insieme dell'analogia fra il Sole e il bene, e un'illustrazione dettagliata dei termini che vi sono implicati, cfr. CALABI 2003. 
come suo oggetto di conoscenza, ma non coincidente con esso ${ }^{7}$. Questo rapporto proporzionale, fin qui istituito dal punto di vista epistemologico della conoscenza e della conoscibilità dei suoi termini, appare immediatamente ampliato anche all'ambito ontologico dell'essere e dell'essenza, perché Socrate riconduce pure al Sole, «che certo

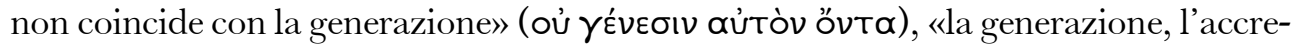

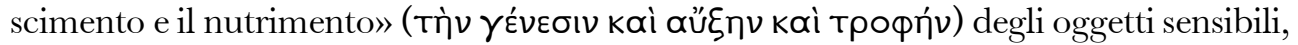
il che porta ad attribuire di conseguenza ${ }^{8}$, appunto per mantenere l'identità di rapporto sui due piani, la stessa funzione al bene rispetto agli intellegibili, che dal bene, «che non consiste propriamente in un'essenza» (oủk oúoías ővTos), devono dunque

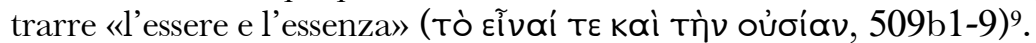

La pur sintetica esposizione di una sezione così complessa e densa della Repubblica restituisce senza dubbio un significato dell'aggettivo ơvó $\lambda \circ \gamma \circ$, , del resto comune nella lingua greca, come "proporzione" o "identità di rapporto" funzionale fra termini situati, in tal caso, in ambiti di per sé eterogenei, l’intellegibile e il sensibile, fra i quali pare sussistere tuttavia una relazione di somiglianza cui è associata un'azione

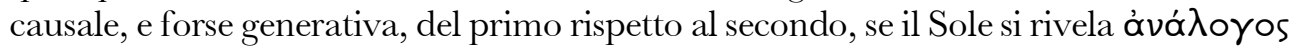

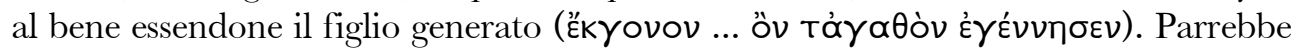
quindi che al rapporto di "analogia” fra il Sole e il bene si accompagni una relazione di somiglianza, come si dirà successivamente nel linguaggio della scolastica, dell'“analogo" all"“analogato", cui non è forse estranea una forma di derivazione del primo dal secondo; ciò suggerisce un supplemento di analisi di questo passo, che sembra introdurre una nozione sensibilmente diversa, e filosoficamente più densa, di "analogia” rispetto al significato comune di "proporzione”. Innanzitutto, va notato che Socrate stabilisce l'analogia fra il Sole e il bene per ovviare a quella che dichiara essere la sua, vera o presunta, ignoranza intorno al bene in sé, in modo da limitarsi a esporre,

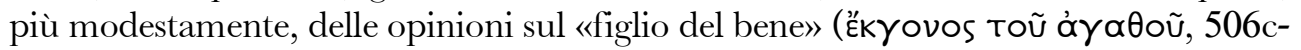
e); ma nulla impedisce in linea di principio che del bene in sé si abbia conoscenza

${ }^{7}$ Lo stesso rapporto proporzionale che lega il bene, l'intelletto e gli intellegibili nell'intellegibile e il Sole, la vista e gli oggetti visibili nel sensibile si trova ribadito poco oltre, in 508e5-509a4, ed esteso anche alla conoscenza intellettuale e alla facoltà della visione: «Ma come sul piano visibile la luce e la vista possono essere correttamente considerate simili al sole, ma non è corretto ritenere che siano il sole, così su quest'altro piano è corretto considerare che scienza e verità siano entrambe simili al bene, ma non è corretto ritenere che l'una o l'altra di esse sia il bene».

${ }^{8}$ Cfr. Repubblica VI 509b1-6: «Dirai, credo, che il Sole non solo procura agli oggetti visibili la facoltà di essere visti, ma anche la generazione, l'accrescimento e il nutrimento ... (...) Pertanto, dirai anche (kaì ... Toívuv) che agli oggetti di conoscenza non deriva dal bene solo l'essere conosciuti, ma che essi ne traggono l'essere e l'essenza ...». Appare chiaro come l'attribuzione al bene del potere di conferire l'essere e l'essenza agli intellegibili, sul piano ontologico, sia il risultato dell'applicazione dell'analogia, appunto nella misura in cui tale potere deve appartenere anche al bene in quanto è stato attribuito al Sole rispetto agli oggetti sensibili.

${ }^{9}$ Difficoltà e limiti dell'analogia sono segnalati da VEGETTI 2003, pp. 269-273. 
piena e diretta, cioè indipendentemente dal suo "analogo", se anzi questo deve essere appunto il caso dei filosofi governanti (506a). Il ricorso all'analogia non è dunque giustificato dall'esigenza di giungere alla conoscenza di un principio di per sé direttamente inconoscibile a partire dai suoi prodotti, come avviene invece per l'analogia dell'essere nella tradizione scolastica.

Ma veniamo al punto centrale: in che senso il Sole è detto Ékyovos e ơvád rispetto al bene? Abbiamo constatato come, in 508b-c, il Sole si riveli "figlio" del bene appunto nella misura in cui adempie, nel mondo sensibile, allo stesso tipo di funzione

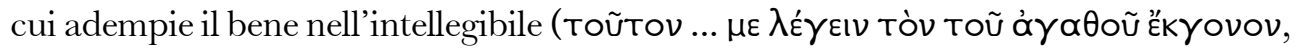
508b12-13), cioè quella di permettere, tramite la luce che emette, alla vista di vedere e agli oggetti visibili di essere visti, con l'ulteriore riconoscimento di una somiglianza fra il Sole, la luce e la vista, che pure non coincidono fra loro, anche se il Sole può essere oggetto di visione da parte della vista; più avanti (509b), tale funzione del Sole finisce per coinvolgere la generazione e l'alimentazione delle cose sensibili. Allo stesso modo, nell'intellegibile, il bene rende possibile, tramite la verità che procura, all'intelletto di conoscere e agli intellegibili di essere conosciuti, sussistendo ancora una volta una somiglianza fra il bene, la verità e l'intelletto, che pure, nuovamente, non coincidono fra loro, benché il bene possa essere oggetto di conoscenza da parte dell'intelletto (508d-509a); più avanti (509b), anche al bene si attribuisce la facoltà di fornire agli intellegibili l'essere e l'essenza. La "filiazione" del Sole dal bene si traduce pertanto in una relazione analogica fra il bene e il Sole, suo "figlio" e suo "analogo" - i due termini risultando qui senza alcun dubbio sinonimi -, che rimane, in tutta la sua estensione, puramente funzionale: quanto l'analogia stabilisce è infatti un'identità di rapporto fra il bene, l'intelletto e gli intellegibili, da una parte, e fra il Sole, la vista e gli oggetti visibili, dall'altro. È importante ribadirlo: ciò che è identico è il rapporto proporzionale fra i termini posti sui due diversi piani, ma da questo nulla si ricava né si può ricavare rispetto alle modalità specifiche di tale rapporto, nell'intellegibile e nel sensibile. Il bene è causa di conoscenza, conoscibilità ed essere, rispettivamente, per l'intelletto e per gli intellegibili, come il Sole è causa di visione, visibilità e generazione, rispettivamente, per la vista e per gli oggetti visibili, ma non certamente allo stesso titolo e nello stesso modo: infatti, se del Sole è lecito sostenere che è causa di visione tramite la sua luce e di generazione tramite il suo calore e, parallelamente, del bene è altrettanto lecito ritenere che è causa di conoscenza ed essere tramite la verità e l'essere che procura, è però del tutto evidente che l'analogia riguarda unicamente l'identità di rapporto proporzionale che il Sole e il bene intrattengono con la vista e gli oggetti visibili e con l'intelletto e gli oggetti intellegibili, ossia un'identità funzionale del Sole e del bene, ma non fornisce nessuna informazione intorno ai modi delle rispettive funzioni, perché, anzi, se si prendono in considerazione i modi delle relazioni fra il bene, l'intelletto e gli intellegibili e fra il Sole, la vista e gli oggetti visibili, l'analogia vacilla. Appare del resto immediatamente chiaro che, mentre per esempio la luce del Sole è 
esclusivamente condizione di visione (per la vista) e di visibilità (per gli oggetti visibili), ma non costituisce né il contenuto della visione, che è il colore, né l'elemento visibile degli oggetti visibili, che è, di nuovo, il colore $(507 \mathrm{~d})$, la verità conferita dal bene si pone invece non solo come condizione di conoscenza (per l'intelletto) e di conoscibilità (per gli intellegibili), ma anche come il contenuto proprio della conoscenza intellettuale e come l'elemento propriamente conoscibile degli oggetti intellegibili (508d): in altre parole, dal punto di vista di Platone, senza la luce non si danno visione né visibilità nel mondo sensibile, ma ciò che la visione vede e ciò che è visto da essa è il colore, laddove senza la verità non si danno conoscenza né conoscibilità nell'intellegibile, perché ciò che la conoscenza conosce e ciò che è conosciuto da essa è appunto la verità stessa ${ }^{10}$. Nell'analogia fra il Sole e il bene non sembra dunque che si prefiguri fin qui alcun genere di relazione che vada oltre l'identità di rapporto caratteristica della proporzione matematica né essa è tale da garantire, attraverso l'esame dello statuto ontologico e dei modi funzionali dell'“analogo" (il Sole), una qualche conoscenza dello statuto ontologico e dei modi funzionali dell'“analogato" (il bene) ${ }^{11}$.

Ma, come già sappiamo, vi è di più, giacché Socrate fornisce un elemento aggiuntivo che suggerisce forse di concepire l'analogia come un legame assai più stretto e incisivo di un'identità di rapporto proporzionale, se è vero che il Sole è non solo «il figlio del bene», tale "filiazione" manifestando unicamente, lo si è visto, un'identità funzionale a un livello inferiore, ma anche che è «a esso assai simile» (ómoiótatos

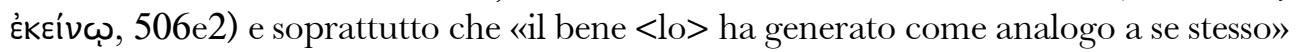

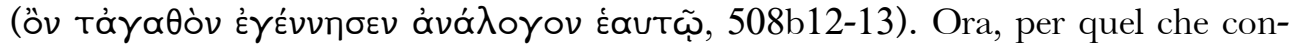

${ }^{10}$ Come è noto, la verità è innanzitutto, secondo Platone, un carattere ontologico delle cose che sono, che consiste nel loro grado di stabilità e immutabilità. In questa misura, la verità rappresenta propriamente ciò che l'intelletto conosce e il tratto conoscibile dell'oggetto conosciuto. Ciò suggerisce a mio avviso di tornare sulla traduzione di 508d, di cui non mi pare si possa accogliere la resa più diffusa: «quando

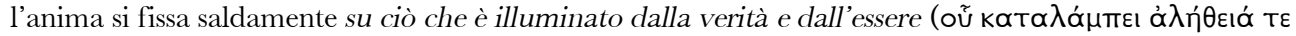
kaì tò öv), allora lo pensa e lo conosce ...», in base alla quale la verità e l'essere si porrebbero soltanto come condizioni di conoscibilità degli oggetti conosciuti in se stessi (come nel caso della luce che permette di vedere i colori), con l'imbarazzante conseguenza che le idee intellegibili (= l'essere) risulterebbero conoscibili in quanto illuminate dalla verità e dall' essere. Credo perciò sia più opportuno intendere il verbo

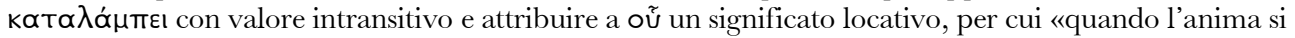
fissa saldamente là dove risplendono la verità e l'essere, allora pensa e conosce il suo oggetto ...», in quanto esso coincide appunto con la verità e l'essere.

${ }^{11}$ Non posso perciò condividere la posizione difesa da DiXSAUT 2000, pp. 126 ss.: « «.. soleil, lumière, vision ne sont pas seulement des termes présentant des rapports analogues à ceux qui, dans le domaine de la connaissance, relient les termes correspondants, ce sont des termes qui ressemblent, et peut-être les seules en qui puisse se dire ce que font les termes qui leur correspondent». Sottolinea invece assai opportunamente il carattere funzionale dell'analogia fra il Sole e il bene, e l'impossibilità di trarre da essa un'indicazione pienamente coerente dei modi funzionali in essa implicati, F. FERRARI 2003b, pp. 295303. 
cerne la "somiglianza", essa è esplicitamente introdotta in parallelo con la "filia-

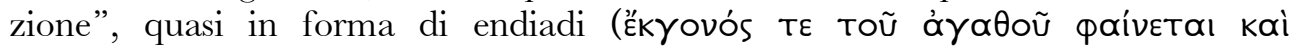

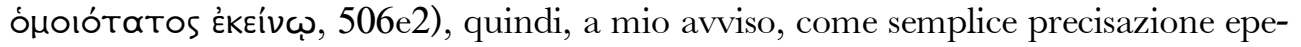
segetica che in nulla interviene a modificare natura e struttura dell'analogia. Diverso è il caso dell'affermazione della generazione del Sole da parte del bene che, per quanto si riveli indubbiamente assai più impegnativa e gravida di conseguenze, non impone però neanch'essa immediatamente che il Sole sia figlio del bene perché il bene lo ha generato come suo analogo né che sia analogo al bene perché il bene lo ha generato come tale, ossia che, in altre parole, "filiazione" e "generazione" risultino reciprocamente implicate nell'analogia. Beninteso, il termine ékyovos evoca certamente il "figlio" in quanto generato o prodotto dal "padre", ma credo di aver mostrato che tale riferimento allude di fatto, nel nostro passo, soltanto all'identità funzionale del "figlio" rispetto al "padre", sicché il "figlio" è detto generato o prodotto dal padre appunto nella misura in cui ne replica la funzione; come pure la "somiglianza" (ómoı́tns), che può indicare senz'altro in assoluto, nella riflessione di Platone, un nesso ontologico che comporta una condivisione di essere, come avviene precisamente

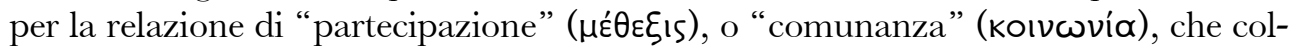
lega il sensibile all'intellegibile fissandone il vincolo causale ${ }^{12}$, ma che pare assumere qui un carattere esclusivamente funzionale, se, nuovamente, il Sole è ómoiótatos al bene essenzialmente rispetto all'esercizio della sua funzione.

Resta tuttavia che, nonostante simili precisazioni e limitazioni, Socrate dichiara

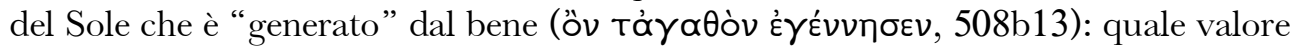
attribuire a tale affermazione? Suggerisco di intendere la "generazione" del Sole dal bene, nel libro VI della Repubblica, sulla base di una sequenza argomentativa di questo genere (che riproduce d'altra parte fedelmente l'ordine e lo sviluppo del discorso di Socrate da 506d a 508c): non "poiché il bene ha generato il Sole, allora il Sole ne è in questa misura il figlio, perciò simile a esso e suo analogo, che adempie alla stessa funzione del bene, a un livello inferiore, in quanto conserva una traccia sostanziale e funzionale della sua derivazione dal bene"; bensì "poiché il sole adempie alla stessa funzione del bene, a un livello inferiore, allora ne è in questa misura un analogo, cioè come un figlio, simile al padre e da esso generato". In altre parole, la duplicazione funzionale, a un livello inferiore, suggerisce a Socrate l'immagine del "figlio", come "duplicato" che riproduce l'azione del padre in un ambito eterogeneo e minore, che a sua volta introduce una relazione di somiglianza, del "figlio" al "padre" di cui agisce come "duplicato", e un rapporto di derivazione o generazione, del "figlio" dal "pa-

\footnotetext{
${ }^{12}$ Sia lecito rinviare su questo punto, per un esame della cruciale nozione di "partecipazione" nel pensiero di Platone, con discussione della bibliografia pertinente, a FRONTEROTTA 2001, specie pp. 148$151,195-222$ e $280-287$.
} 
dre" di cui è appunto "figlio", la generazione costituendo, in questa prospettiva, l'ultimo anello di una catena metaforica che non incide a mio avviso sulla natura semplicemente funzionale dell'analogia ${ }^{13}$. La lettura da me appena proposta, a prima vista alquanto congetturale se non perfino capziosa, si appoggia invece su alcune considerazioni di fondo, secondo me decisive, che rivelano l'insormontabile difficoltà che sorgerebbe, in un contesto platonico, a concepire l'esistenza del Sole nel sensibile, e la sua generazione dal bene, come l'esito di un'azione causale o di un atto derivativo reali, quindi tali da violare i limiti solo funzionali dell'analogia in favore di una ben più impegnativa analogia ontologica fra il Sole e il bene.

In primo luogo, infatti, se è indubbio che Platone pone una relazione causale assolutamente reale fra il sensibile e l'intellegibile, appunto la "partecipazione" già evocata sopra, tramite la quale le cose sensibili acquistano dalle idee intellegibili l'essenza, le proprietà e il nome riconosciuti loro nell'esperienza comune, è evidente che non si tratta in nessun caso di ammettere una generazione o una derivazione integrale delle cose sensibili partecipanti dalle corrispondenti idee partecipate, e ciò per l'ottima ragione che si giungerebbe così a svuotare il sensibile di ogni sostanzialità, sottraendogli lo statuto suo proprio di realtà esistente (certamente esistente, anche se a un livello diverso e inferiore a quello delle idee), riducendolo così, in sé e per sé, al puro nulla, alla semplice assenza dell'essere, e determinando perciò una prospettiva ontologica su un solo livello, quello delle idee intellegibili che, esercitando nel vuoto la propria azione causale, producono a certe condizioni delle imitazioni, copie o parvenze di se stesse, sussistenti solo in virtù di quelle. Una concezione del genere mi sembra smentita nettamente, fra l'altro, dalla constatazione che, anche quando viene sottolineata l'assoluta "minorità" ontologica delle cose sensibili rispetto alle idee, nessun passo dei dialoghi giunge mai a sancirne l'effettiva coincidenza con il non essere e con il puro nulla. Anzi, nel libro V della Repubblica (478e-479d), l'unico passo effettivamente esplicito in proposito, viene stabilito con chiarezza che la sfera sensibile occupa di per

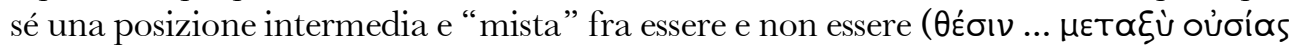

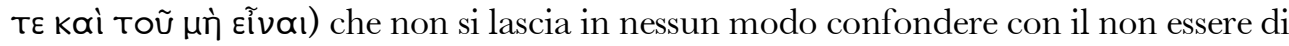
ciò che non è affatto, né dal punto di vista ontologico né dal punto di vista epistemologico del genere di conoscenza che le si addice. Platone tiene dunque ferma quella radicale duplicità ontologica caratteristica della sua prospettiva filosofica bipolare ed ecco perché occorre distinguere due "mondi" o almeno due piani del reale del tutto irriducibili l'uno all'altro e perciò, anche se a diverso titolo e con un diverso grado ontologico, entrambi sostanziali ${ }^{14}$. Nella stessa direzione va l'introduzione della $\chi \omega ́ p \alpha$

${ }^{13}$ Un'ampia discussione dei diversi termini impiegati per indicare la relazione, solo funzionale o propriamente derivativa, del Sole con il bene e un acuto bilancio critico intorno al tema dell'effettiva generazione del Sole dal bene sono forniti da CALABI 2003, pp. 331-336 e 351-354.

${ }^{14} \mathrm{Si}$ veda ancora, in proposito, FronterotTa 2001, pp. 151-154; cfr. pure FronterotTa 2006, pp. 412-420. 
nel Timeo, con il suo statuto ontologico e funzionale di principio coeterno alle idee intellegibili e di sostrato "spazio-materiale" delle cose sensibili, che produce propriamente e suscita da sé come enti (pur parzialmente) sostanziali in virtù delle modificazioni della sua configurazione che si determinano in conformità alle idee intellegibili,

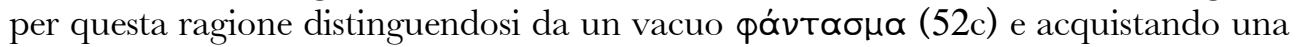
dimensione sostanziale che deve senza dubbio la sua forma e la sua determinazione alle idee intellegibili di cui è riproduzione o imitazione, ma che, anche indipendentemente da tale forma e determinazione, non si ridurrebbe al nulla assoluto, ma confluirebbe nuovamente nella $\chi \omega ́ \rho \alpha$, nel sostrato "spazio-materiale" informe che è sempre e non si corrompe, che è, si, opposto al modello intellegibile quanto alla sua pura indeterminazione, ma complementare a esso dal punto di vista ontologico. $\mathrm{Si}$ deve riconoscere così, nuovamente, un modello onto-cosmologico almeno dualista, che nel Timeo si rivela particolarmente evidente, basato cioè sull'irriducibilità di due principi sostanziali antagonisti, il modello intellegibile e la $\chi \omega ́ p \alpha$, fra i quali è stabilita una relazione, espressa nel dialogo tramite la metafora "artigianale" del demiurgo che dispone della $\chi \omega ́ p \alpha$ come di un materiale informe, che egli lavora manualmente, conferendogli una figura, un ordine e una struttura che appartengono originariamente al modello intellegibile e che egli può, dopo averli contemplati nel modello intellegibile, riprodurre nella copia sensibile ${ }^{15}$. Tornando al problema in esame, mi pare insomma che una simile considerazione opponga un argomento irresistibile alla possibilità di intendere il rapporto fra il Sole, nel sensibile, e il bene, nell'intellegibile, in forma propriamente generativa o derivativa, inducendo piuttosto a ipotizzare fra i due piani una relazione causale di qualche genere, che Socrate lascia tuttavia qui inesplicata ${ }^{16}$.

Ma in secondo luogo, e soprattutto, Platone non associa mai, né potrebbe comunque associare, a questa relazione causale fra il sensibile e l'intellegibile (o fra il Sole e l'idea del bene) la nozione di analogia, che abbiamo visto non esprimere altro che un'identità di rapporto o di funzione, orizzontale o verticale, fra termini appartenenti ad ambiti anche eterogenei, e ciò nella misura in cui la relazione causale fra i due piani del reale implica una ben più impegnativa comunanza ontologica che rimane estranea alla definizione e all'impiego dell'analogia, benché sia naturalmente del tutto plausibile che, stante la relazione di dipendenza causale del sensibile dall'intellegibile, ogni stato di cose, e dunque ogni genere di rapporto, anche analogico, fra termini appartenenti all'ambito sensibile o, distributivamente, all'ambito sensibile e all'ambito intellegibile, sia in qualche modo connesso a tale relazione. Si può inoltre attribuire a Platone un argomento supplementare contro l'associazione della nozione di analogia alla relazione causale fra il sensibile e l'intellegibile, vale a dire il fatto che non occorre

\footnotetext{
${ }^{15}$ Cfr. in ultimo, su questa forma di "dualismo" che caratterizza la prospettiva onto-cosmologica del Timeo, Fronterotta 2014.

${ }^{16}$ Si veda in questo senso l'esemplare conclusione di VEGETTI 2003, p. 269.
} 
introdurre uno strumento apposito - appunto l'analogia - per illustrare i termini di questa relazione, e risalire così, in virtù della relazione stessa, alla conoscenza della causa intellegibile a partire dal suo effetto sensibile, perché l'ambito dell'intellegibile appare di per sé, nella riflessione di Platone, pienamente conoscibile in tutte le sue regioni e in tutte le sue parti, compreso il bene, che è infatti successivamente descritto, nel libro VI della Repubblica, come oggetto di $\mu$ á́ $\theta \eta \mu \alpha$ (504e4), cioè di una conoscenza, per quanto somma, però accessibile, quindi come sapere concretamente posseduto dai filosofi governanti (506a), infine come realtà che, nonostante la sua asso-

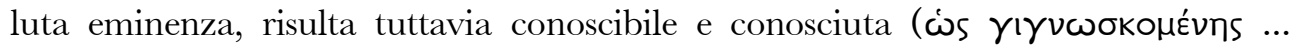
Sıavooũ, 508e3). Come è ben messo in evidenza, per esempio, dalla dottrina della reminiscenza, la relazione causale fra il sensibile e l'intellegibile è infatti sufficiente a garantire la presenza, nel sensibile, di "tracce" dell'intellegibile, a partire dalle quali si rende senza dubbio possibile giungere alla piena conoscenza delle idee, che si configura precisamente, per ricorrere alla formulazione del Fedro, come un movimento ascendente dalla molteplicità del sensibile verso l'unità colta con il ragionamento, cioè

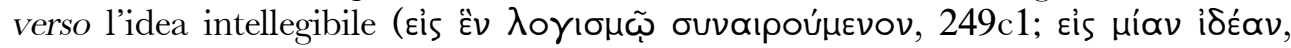
$265 \mathrm{~d} 3)^{17}$ - e lo strumento dell'analogia può intervenire in questo contesto limitatamente all'esigenza di stabilire dei rapporti funzionali fra i due piani, prima o indipendentemente dall'effettiva conoscenza di essi ${ }^{18}$; mentre non si tratta certamente di concepire le cose sensibili come prodotti generati o integralmente derivati dalle idee intellegibili, per ottenere, attraverso la conoscenza di essi, un parziale accesso alle idee intellegibili, da considerare inoltre, in tal caso, di per sé inconoscibili, in base a un movimento discendente per cui la conoscenza dell'intellegibile si fonda soltanto sulla conoscenza del sensibile in quanto generato o derivato a partire dall'unità intellegibile, cioè secondo un rapporto ợ $\varphi^{\prime} \varepsilon$ vós - e solo a queste condizioni lo strumento dell'analogia potrebbe assumere i tratti che gli sono riconosciuti nella tradizione scolastica,

\footnotetext{
${ }^{17}$ Introdotta nel Menone (81a-86c), quindi più ampiamente illustrata nel Fedone (74a-76a) e nel Fedro (246e-249d), la dottrina della reminiscenza fa coincidere la conoscenza con il ricordo di ciò che si è già appreso in un tempo precedente $\mathrm{o}$, più esattamente, di ciò che l'anima immortale ha appreso quando, prima di fare ingresso nel corpo mortale, rivolgeva il suo sguardo alle realtà intellegibili. Ogni volta che entra in un corpo, l'anima dimentica ciò che ha appreso, per ricordarsene in seguito, nel corso della vita mortale, in virtù della percezione delle cose sensibili la ricerca della verità consistendo precisamente nel recupero di tale ricordo. Si veda solo in proposito, sulla reminiscenza nel suo insieme e sul suo significato filosofico, SCOTT 1995.

${ }^{18}$ In altre parole, se nel libro VI della Repubblica Socrate dicesse cosa sia il bene e quali le sue relazioni con l'intelletto e gli intellegibili, non occorrerebbe stabilire nessuna analogia con il figlio del bene, con il Sole. Il fatto che si riconosca che tale conoscenza è possibile, e appartiene effettivamente ai filosofi, indica che l'analogia può essere utile solo nel caso in cui essa sia, per qualche motivo, attualmente indisponibile.
} 
nella forma di una relazione che, in virtù dell'assunto della generazione o della derivazione del sensibile a partire dall'intellegibile, permetterebbe di trarre dalla conoscenza del sensibile una conoscenza parziale e indiretta dell'intellegibile.

Mi pare che un esito così estremo sia raggiunto piuttosto, mutatis mutandis, nel corso della tradizione platonica antica, la cui trattazione oltrepassa gli obiettivi del mio contributo e che richiamerò soltanto, come pura suggestione, a mo' di conclusione. La prima tappa di questo processo è rappresentata verosimilmente dall'identificazione, diffusa fra gli autori medioplatonici tra I e II secolo d.C., dell'idea del bene della Repubblica con il demiurgo cosmico del Timeo, variamente concepito come una divinità plurale che esercita competenze intellettuali e produttive ${ }^{19}$; ma è notoriamente Plotino a compiere la decisiva mossa esegetica, collocando l'esposizione platonica dell'idea del bene in parallelo con la discussione intorno all'uno oltre l'essere contenuta nella seconda parte del Parmenide, e così individuando un principio primo del reale, appunto l'uno o il bene, che trascende l'oúoía intellegibile e che si pone perciò al di là dell'essere, della conoscenza e del discorso, in una condizione di assoluta inattingibilità che può essere solo indirettamente avvicinata a partire dai suoi prodotti, che, pur restandogli inferiori, conservano tuttavia un'impronta della sua potenza generatrice. Si comprenderà come, in una interpretazione che trasforma il dualismo platonico in un monismo radicale, facendo dell'idea del bene della Repubblica un principio primo che genera tutte le cose, l'unico accesso possibile a tale principio assolutamente trascendente consista nella conoscenza dei suoi prodotti, innanzitutto, per attenersi all'immagine della Repubblica, a partire dal Sole, la cui analogia con il bene assume necessariamente i tratti di una relazione derivativa integrale ớ ф’évós, che procede verticalmente attraverso livelli successivi di esistenza e di realtà la cui omogeneità è garantita dalla comune provenienza e generazione ${ }^{20}$.

${ }^{19}$ Questa è senza dubbio la posizione di Attico e di Plutarco, ma anche Alcinoo e Numenio identificano l'idea del bene con la divinità, nella quale distinguono però un livello puramente contemplativo (effettivamente coincidente con l'idea del bene, di cui per esempio Numenio, fr. 16 des Places, afferma

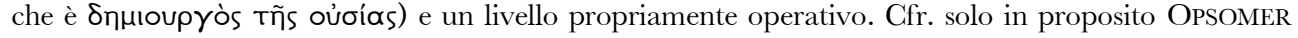
2005 e FERRARI 2015, specie pp. 325-333.

${ }^{20}$ È impossibile riportare o anche solo citare i numerosissimi passi che testimoniano dell'articolata operazione esegetica che Plotino compie nella sua lettura del testo platonico; mi limito dunque a indicarne tre fra i più celebri: Enneadi V 1 (10) 8; VI 7 (38) 16; e VI 9 (9) 3-6. Nel secondo di essi, in particolare, Plotino si sofferma precisamente sull'analogia fra il Sole e il bene, attribuendo a quest'ultimo il potere di produrre, insieme con la realtà intellegibile nel suo complesso, anche la "luce" intellegibile necessaria a conoscerla, esattamente come il Sole che produce, insieme con la realtà sensibile nel suo complesso, anche la "luce" sensibile necessaria a vederla, sicché l'analogia è qui strettamente connessa alla derivazione dal bene, fino a consistere in tale derivazione, perché il Sole riproduce a un livello inferiore la stessa azione del bene in quanto da esso deriva e ne è una traccia. Vastissima è pure la letteratura critica su questo punto: ricorderò soltanto perciò, con una scelta tanto parziale quanto arbitraria, il seminal paper di DODDs 1928; quindi l'opera fondamentale di BEIERWALTES 1985; infine il penetrante 


\section{Bibliografia}

Abbate, M. (2003), «Il Bene nell’interpretazione di Plotino e di Proclo», in M. Vegetti (ed.), Platone, La Repubblica, V, Libri VI-VII, Napoli, 625-678.

Aubenque, P. (1978), «Les origines de la doctrine de l'analogie de l'être. Sur l'histoire d'un contresens», Les Etudes Philosophiques, 103, 2-13.

Aubenque, P. (2013), Le problème de l'être chez Aristote. Essai sur la problématique aristotélicienne, Paris $1962\left(2013^{6}\right)$.

Beierwaltes, W. (1985), Denken des Einen. Studien zur neuplatonischen Philosophie und ihrer Wirkungsgeschichte, Frankfurt am Mein.

Berti, E. (1984), «L'analogia dell'essere nella tradizione aristotelico-tomista», in Metafore dell'invisibile. Ricerche sull'analogia, Contributi al XXXlll Convegno del Centro di Studi filosofici di Gallarate, Brescia, 13-33.

Berti, E. (1987), «L'analogia in Aristotele. Interpretazioni recenti e possibili sviluppi», in G. Casetta (ed.), Origini e sviluppi dell'analogia, Roma, 94-115.

Calabi, F. (2003), «Il sole e la sua luce», in M. Vegetti (ed.), Platone, La Repubblica, V, Libri VI-VII, Napoli, 327-354.

Courtine, J.-F. (2005), Inventio analogiae. Métaphysique et onto-théologie, Paris.

Dixsaut, M. (2000), «L'analogie intenable: le Soleil et le Bien», in Platon et la question de la pensée. Études platoniciennes I, Paris, 121-151.

Dodds, E.R. (1928), «The Parmenides of Plato and the origin of the neoplatonic One», Classical Quarterly, 22, 129-142.

Ferber, R. (2015), Platos Idee des Guten, Sankt Augustin 2015².

Ferrari, F. (2003a), «Conoscenza e opinione: il filosofo e la città», in M. Vegetti (ed.), Platone, La Repubblica, IV, Libro V, Napoli 2003, 393-419.

Ferrari, F. (2003b), «L'idea del bene: collocazione ontologica e funzione causale», in M. Vegetti (ed.), Platone, La Repubblica, V, Libri VI-VII, Napoli, 287-325.

Ferrari, F. (2015), «Metafisica e teologia nel medioplatonismo», Rivista di Storia della filosofia, LXX, 321-337.

Fronterotta, F. (2001), Methexis. La teoria platonica delle idee e la partecipazione delle cose empiriche. Dai dialoghi giovanili al Parmenide, Pisa.

Fronterotta, F. (2006), «Questioni eidetiche in Platone: il sensibile e il demiurgo, l'essere e il bene», Giornale critico della filosofia italiana, LXXXV 3, 412-436.

Fronterotta, F. (2007), «EINAI, OY $\Sigma$ IA e ON nei libri centrali della Repubblica», in F.L. Lisi (ed.), The Ascent to the Good, Sankt Augustin, 115-160.

Fronterotta, F. (2014), «Modello, copia, ricettacolo: monismo, dualismo o triade di principi nel Timeo?», Méthexis, XXVII, 95-118.

Fronterotta, F. (2016), «ANA $О Г І$ IA in Platone: occorrenze e significato», Archivio di Filosofia, LXXXIV, 49-64.

saggio di SzLEZÁK 1979. Ampia discussione, con riferimento alla bibliografia pertinente, in ABBATE 2003. 
Krämer, H.-J. (1967), Der Ursprung der Geistmetaphysik. Untersuchungen zur Geschichte des Platonismus zwischen Platon und Plotin, Amsterdam $1967^{2}$.

Lyttkens, H. (1952), The Analogy between God and the world: an investigation of its background and interpretation of its use by Thomas of Aquino, Uppsala.

Melchiorre, V. (1996), La via analogica, Milano.

Opsomer, J. (2005), «Demiurge in Early Imperial Platonism», in R. Hirsch-Luipold (hrsg.), Gott und die Götter bei Plutarch. Götterbilder-Gottesbilder-Weltbilder, Berlin-New York, 51-99.

Scott, D. (1995), Recollection and experience. Plato's theory of learning and its successors, Cambridge.

Szlezák, Th.A. (1979), Platon und Aristoteles in der Nuslehre Plotins, Basel-Stuttgart.

Vegetti, M. (2003), «Meghiston mathema. L’idea del "buono” e le sue funzioni», in M. Vegetti (ed.), Platone, La Repubblica, V, Libri VI-VII, Napoli, 253-286. 